\title{
The Integrable System on the Second-Order Eigenvalue Problem with the Potential and Speed
}

\author{
Sun Yinghui ${ }^{1, *}$ and Li Zihua ${ }^{2}$ \\ ${ }^{1}$ School of Material Science and Engineering, Tianjin University, Tianjin, China \\ ${ }^{2}$ Applied Mathematics, Hebei University of Technology, Tianjin, China
}

Received: 6 Feb. 2013, Revised: 7 Jun. 2013, Accepted: 8 Jun. 2013

Published online: 1 Sep. 2013

\begin{abstract}
In this paper, the Bargmann system for the second-order eigenvalue problem with the energy is dependent on potential and speed $L \varphi-\left(\partial^{2}+\lambda u+v\right) \varphi-\lambda \varphi_{x}$ is discussed.

First of all, some basic concepts are introduced. After that, by means of the auxiliary problem and the isospectral compatible condition, the reasonable bi-Hamilton operator $\mathrm{K}$ and $\mathrm{J}$ are defined, and the evolution equations related to the spectral problem are obtained. Using the functional gradient and Lenard recursive sequence, the Bargmann constraint is given. By the constraint relation between the potential function and the eigenvector, the associated Lax pairs are nonlineared, then the Bargmann system of the eigenvalue problem is found. According to the viewpoint of Hamilton mechanics, a reasonable coordinate system has been found. The Bargmann system is transformed into the Hamilton canonical equations in the coordinate system. Finally, based on the Liouville theorem and confocal involutive system, the integrability of the Hamilton system is proved.
\end{abstract}

A new integrable system is found. Moreover, the involutive representations of the solutions for the evolution equations are generated.

Keywords: Eigenvalue problem, potential, Bargmann system, non-lineared Lax pairs, Hamilton integrable system, involutive representation.

\section{Introduction}

\subsection{Soliton's history of development and research significance}

Soliton is one of the non-linear phenomenon which can be produced in the laboratory and first observed in nature. It has been more than one hundred years from discovering soliton to now, but it's great development and application in many subjects (such as optical fiber communication, nonlinear optics space soliton, flux son device, the biology DaWeiDuo husband soliton, plasma of solitary wave, etc.) began in $1970 \mathrm{~s}$. Below we outline the vital events in its development process, as well as its research significance and status quo.

The first time people has observed soliton which is exactly recorded is that one day in August 1834, when Russell riding on the bank of river, he acutely found that the column aroused by the bow was a very unusual natural phenomenon. As a successful scientist, a essential quality is that he has the ability to be aware of the essentially different new phenomenon from the complicated phenomenon, and Russell had the ability. After discovering the translation wave (now we called it a solitary wave), he spent a lot of time doing experiment to determine the nature of this wave. But he has not been theoretically confirmed the existence of solitary wave.

Throughout the history of science, it is not difficult to find that putting forward a new idea or introducing a new concept often can cause long periods of intense debate, sometimes even suspected and animadvert. Solitary wave fate is also so. The argument until 1895, Korteweg and DE Vries attained shallow water wave equation from fluid mechanics research. Meanwhile, they also got the equation of travelling wave solutions, it describes the solitary wave motion found by Russell in the wavelength tending to infinite cases.

The presentation of $\mathrm{KdV}$ equation theoretically expounded the existence of solitary wave. But the academic circles haven't been able to answer whether solitary wave is stable or not. Until 1965, the American mathematician Kruskal and Zabusky made calculation in

\footnotetext{
*Corresponding author e-mail: 04guojizhengzhi@ sina.com
} 
computer describing the whole process of the collision of KdV equation's two wave. First of all, solitary wave maintained same in the collision like "through Ming" through each other. Secondly, solitary wave occurred phase shifting after the collision. Since then, people admitted that solitary wave is stable, after the collision two or more solitary wave kept each of the same waveform and speed to move on propagation. The problem with solitary wave revealed by solid state physics, nonlinear electromagnetism and neural dynamics encouraged people to consider the area beyond fluid, whether the solitary wave existed. These problems caused the people's extensive concern.

The recent development of the soliton has made more great progress than before, rough summarized as: from one dimension to multidimensional; By single soliton evolution party ride to the coupling evolution equations; From classic to the quantum; From the single subject to interdisciplinary. The study was critical for the development of soliton theory. When the development of the theoretical research on the technology had great application prospects, production and technology need to promote the development of the theory in a further degree. With the coming of information society, information increased rapidly, transferring and processing information rapidly not only had an important role on production, scientific research but also had a far-reaching influence on the whole social's progress.

\subsection{Integrable system research significance and present situation}

Integrable system is a challenging research topic in soliton theory which has draw the attention of many scholars at home and abroad. The "nonlinear science" leaded by $\mathrm{Gu}$ Chao hao academician had been listed as our country climbing project, Chinese scholars made a more in-depth and systematic research results in integrable system studying. Recent research shows that integrable system theory is still the mainstream of development of soliton theory at home and abroad and one of the most active research field.

Looking for and expanding integrable system in the meaning of Lax or Liouville [2] is important issue in soliton theory study, and searching for new integrable system and connecting with the equation which has the physical meaning is very difficult. $\mathrm{Bi}$ - Hamilton structure is a direct and beautiful method [3-5] which can prove that nonlinear equation fully integrable. In recent years, Chinese scholars has made a very big achievements in this aspect. The trace identity putted forward by $\mathrm{Tu}$ Guizhang is a effective method of constructing infinite dimensional Hamilton system. Starting by spectral problem, we can can get many equation family and Hamilton system, such as, AKNS, TC, TA, BPT, Yang family $[6,7]$. The Lax nonlinear polarization method put forward by Cao Cewen is a effective method of structuring finite dimensional integrable Hamilton system which can nonlinearized the eigenvalue problem into finite dimensional completely integrable Hamilton system, such as, KdV, AKNS, Jaulent - Miodek, Kaup Newell, clan [8-11], etc. Another important application of this method is transforming the solution of soliton equation connected with eigenvalue problem into into solving compatibility of ordinary differential equation [9-11]. Researching power system's complete integrability in the sense of Liouville, usually puts the system into Hamilton system, it's a good way of the theory of product to study integrability in today.

\subsection{Research content of this paper}

What this article researches is that the second order eigenvalue of energy in accordance with the potential and speed:

$$
L \varphi=\left(\partial^{2}+\lambda u+v\right) \varphi=\lambda \varphi_{x}
$$

$\left(\partial=\frac{\partial}{\partial x}\right.$, characteristic parameter $\lambda \in C$, potential function $u, v$ are $(X, t)$ very value real function). Chapter one, review the soliton and integrable system research significance and status. Chapter two, first introduce some basic concepts and prove some properties. Then work out $\mathrm{X}$ Functional gradient, and take appropriate double Hamilton operator $\mathrm{K}, \mathrm{J}$ and Lenavd recursion sequence $\{j=-1,0,1, \ldots\}$ according to the compatibility conditions, and get the second order eigenvalue problem (1) related development equation family and Lax. Chapter three, first of all get constraint relation and determine nonlinear chemical equation of Lax. Use the Hamilton force System structure characteristics and it's connection to Euler - Lagrange equation. Setting up a set of suitable for this paper differential equation of coordinate system [12] in the symplectic popular $\left(w=\sum_{j=1}^{2}\right.$ $\left.d y_{j} \wedge d z_{j}, R^{2 N}\right) j=i$, so Bargmann system is transformed into equivalent Hamilton canonical system. Chapter four, research Hamilton system through the matrix form, prove the Liouville sense completely integrability, and discussed solution representation of the development of the family of equation. Chapter five, the full text to carry on the summary, the main conclusions are given.

\section{The second chapter second order eigenvalue problem development equation family and Lax said}

In order to study the second order eigenvalue problem, first of all we need to define its basic space.Now, assuming that the second order eigenvalue problem (1) space is the basis of $\Omega$, if the potential function $u, v$ and characteristics as well as their function to $x$ all order derivative are infinite downhill function, the definition of 
$\Omega$ is $(-\infty,+\infty)$, If these functions are cycle for $T$ of the periodic function, the definition $\Omega$ for $[0,2 T]$.

Definition 1. In the problem space into $L_{2}$ inner product operation:

$$
(f, h)_{L_{2}(\Omega)}=\int_{\Omega} f h^{*} d x\langle\infty
$$

Among them: $h^{*}$ stands for the complex conjugate function of $h$.

Definition 2. If profit and profit meet relation:

$$
(A f, h)_{L_{2}(\Omega)}=(f, \bar{A} h)_{L_{2}(\Omega)}
$$

The operator is called the benefit of dual operator. If the profit $=h$, said operator profit for self adjoint operator, otherwise will its known as the self adjoint operator.

Theorem 1. (1) $L=\partial^{2}+\lambda u+v$ for the space of self adjoint operator, and the following pedigree

$$
\left\{\begin{array}{l}
L \varphi=\left(\partial^{2}+\lambda u+v\right) \varphi=\lambda \varphi_{x} \\
\bar{L} \varphi=\left(\partial^{2}+\lambda u+v\right) \psi=-\lambda^{*} \psi_{x}
\end{array}\right.
$$

To form a complete pedigree.

(2) if the eigenvalue problem is (2) characteristic value, then into is real, namely $\lambda=\lambda^{*} \in R$.

Proof. (1) according to the dual operator definition, when

$$
L=\partial^{2}+\lambda u+v
$$

There appears:

$$
\begin{aligned}
(L f, g)_{L_{2}(\Omega)} & =\int_{\Omega}(L f) g^{*} d x \\
& =\int_{\Omega} f\left(\partial^{2}+\lambda u+v\right) g^{*} d x \\
& =\int_{\Omega} f(\bar{L} g)^{*} d x \\
& =(f, \bar{L} g)_{L_{2}}(\Omega)
\end{aligned}
$$

And $L=\bar{L}$, so $L$ for self adjoint operator.

(2) by (2-1) available:

$$
\begin{aligned}
\lambda \int_{\Omega} \varphi_{x} \psi d x & =\int_{\Omega}(L \varphi) \psi d x=\int_{\Omega} \varphi(\bar{L} \psi) d x \\
& =\int_{\Omega}\left(-\varphi \lambda^{*} \psi_{x}\right) d x=\int_{\Omega} \lambda^{*} \varphi_{x} \psi d x \\
& =\lambda^{*} \int_{\Omega} \varphi_{x} \psi d x
\end{aligned}
$$

So $\lambda=\lambda^{*} \in R$.

Theorem 2. Double order eigenvalue problem (2) characteristics function is real function.

Proof. If $\varphi$ is a copmplex function, can make the $\varphi=a+$ $b i$ (including $a, b$ were real function), the $L \varphi=\lambda \varphi_{x}$ in the $x$ can be

$$
\left\{\begin{array}{l}
L a=\lambda a_{x} \\
L a=\lambda b_{x}
\end{array}\right.
$$

Therefore $a, b$ is also type (2) about the characteristic function. To discuss the convenient, can make $\varphi$ is real function.

Theorem 3. If the double order eigenvalue problem (2), the characteristic parameters of the characteristic function, it can get functional gradient for:

$$
\operatorname{grad} \lambda=\left(\begin{array}{l}
\delta \lambda / \delta v \\
\delta \lambda / \delta u
\end{array}\right)=\left(-\int_{\Omega}\left(u \varphi-\varphi_{x}\right) \psi d x\right)^{-1}\left(\begin{array}{c}
\varphi \psi \\
\lambda \varphi \psi
\end{array}\right)
$$

Proof. According to

$$
L=\left.\frac{d}{d \varepsilon}\right|_{\varepsilon=0} L\left(v+\varepsilon \delta v, u+\varepsilon \delta_{u}\right)
$$

From type (= refeq21) get

$$
(L \varphi)^{\cdot}=L^{\prime} \varphi+L \varphi^{\prime}=\left(\lambda \varphi_{x}\right)^{\cdot}=\lambda \cdot \varphi_{x}+\lambda \varphi_{x}
$$

And because of

$$
\begin{aligned}
\int_{\Omega}\left(L \varphi^{\cdot}\right) \psi d x & =\int_{\Omega} \varphi \cdot(\bar{L} \psi) d x=\int_{\Omega}-\varphi \cdot \lambda \psi_{x} d x \\
& =\int_{\Omega} \lambda \varphi_{x}^{\prime} \psi d x
\end{aligned}
$$

Thus

$$
\begin{aligned}
& \int_{\Omega}\left[\left(\partial^{2}+\lambda u+v-\lambda \partial\right) \varphi\right]^{\cdot} \psi d x \\
= & \int_{\Omega}\left[\left(\partial^{2}\right)^{\cdot} \varphi+\partial^{2} \varphi^{\cdot}+(\lambda u)^{\cdot} \varphi+\lambda u \varphi^{\cdot}\right. \\
& \left.+v^{\cdot} \varphi+v \varphi-(\lambda \partial)^{\cdot} \varphi-\lambda \partial \varphi^{\cdot}\right] \psi d x \\
= & \int_{\Omega}\left[(\lambda u)^{\cdot} \varphi+v^{\cdot} \varphi-(\lambda \partial)^{\cdot} \varphi\right] \psi d x \\
= & \int_{\Omega}\left(\lambda \cdot u \varphi+\lambda \delta u \varphi+\delta v \varphi-\lambda \cdot \varphi_{x}\right) \psi d x \\
= & \left.\int_{\Omega}\left[\lambda \cdot\left(u \varphi-\varphi_{x}\right)+(\lambda \delta u \varphi+\delta v \varphi)\right] \psi d x\right] \\
= & 0
\end{aligned}
$$

So we can get functional gradient:

$$
\operatorname{grad} \lambda=\left(\begin{array}{l}
\delta \lambda / \delta v \\
\delta \lambda / \delta u
\end{array}\right)=\left(-\int_{\Omega}\left(u \varphi-\varphi_{x}\right) \psi d x\right)^{-1}\left(\begin{array}{c}
\varphi \psi \\
\lambda \varphi \psi
\end{array}\right)
$$

Is right.

If

$$
\left\{\begin{array}{l}
\varphi_{x x}=\lambda \varphi_{x} \\
\varphi_{t}=\omega_{m} \varphi
\end{array}\right.
$$

Among them:

$$
\left\{\begin{array}{l}
w_{m}=\sum_{j=0}^{m}\left(-\frac{1}{2} b_{j x}-\frac{1}{2} \lambda b_{j}+b_{j} \partial\right) \lambda^{m-j}, m=0,1,2, \cdots \\
g_{j}=\left(\begin{array}{c}
b_{j} \\
b_{j+1}
\end{array}\right), j=-1,0,1, \cdots, m-1 .
\end{array}\right.
$$


The compatibility conditions available:

$$
\begin{aligned}
J & =\left(\begin{array}{cc}
\frac{1}{2} \partial^{3}+v \varphi+\varphi v & 0 \\
0 & \frac{1}{2} \partial
\end{array}\right) \\
K & =\left(\begin{array}{cc}
0 & \frac{1}{2} \partial^{3}+v \varphi+\varphi v \\
\frac{1}{2} \partial^{3}+v \varphi+\varphi v & u \partial+\partial u
\end{array}\right)
\end{aligned}
$$

Theorem 4. Operator $K, J$ are double Hamilton operator, namely to meet skewness, non degenerative, double linear sex, Jacobi equation was established, and meet the relation:

$$
\operatorname{Kgrad} \lambda=J(\lambda \operatorname{grad} \lambda)
$$

Proof. $K, J$ and grad $\lambda$ generation into the type, need to verify

$$
\begin{aligned}
& \left(\begin{array}{cc}
0 & \frac{1}{2} \partial^{3}+v \varphi+\varphi v \\
\frac{1}{2} \partial^{3}+v \varphi+\varphi v & u \partial+\partial u
\end{array}\right)\left(\begin{array}{c}
\varphi \psi \\
\lambda \varphi \psi
\end{array}\right) \\
= & \lambda\left(\begin{array}{cc}
\frac{1}{2} \partial^{3}+v \varphi+\varphi v & 0 \\
0 & \frac{1}{2} \partial
\end{array}\right)\left(\begin{array}{c}
\varphi \psi \\
\lambda \varphi \psi
\end{array}\right)
\end{aligned}
$$

The first line clearly established. The following verification equations on both sides of the second line matrix equal: on the left

$$
\begin{aligned}
= & \left(\frac{1}{2} \partial^{3}+v \varphi+\varphi v\right) \varphi \psi+(\partial u+u \partial) \lambda \varphi \psi \\
= & \frac{1}{2} \varphi_{x x x} \psi+\frac{3}{2} \varphi_{x x} \psi_{x}+\frac{3}{2} \varphi_{x} \psi_{x x}+\frac{1}{2} \varphi \psi_{x x x}+v_{x} \varphi \psi \\
& +2 v \varphi_{x} \psi+2 v \varphi \psi_{x}+\lambda u_{x} \varphi \psi+2 \lambda u \varphi_{x} \psi+2 \lambda u \varphi \psi_{x} \\
= & \frac{1}{2}\left(\lambda^{2} \varphi_{x}-\lambda^{2} u \varphi-\lambda v \varphi-\lambda u_{x} \varphi-\lambda u \varphi_{x}-v_{x} \varphi\right. \\
& \left.-v \varphi_{x}\right) \psi+\frac{3}{2}\left(\lambda \varphi_{x}-\lambda u \varphi-v \varphi\right) \psi_{x}+\frac{3}{2} \varphi_{x}\left(-\lambda \psi_{x}\right. \\
& -\lambda u \psi-v \psi)+\frac{1}{2} \varphi\left(\lambda^{2}\right)
\end{aligned}
$$

$=$ right, so (6) is right.

We can get: $J_{g-1}=0$. And by LenaTd recursion equation

$$
J g_{j}=K g_{j-1}
$$

Can get Lenard recursion sequence $\left\{g_{j} \mid j=-1,0,1, \cdots\right\}$. Note 1. Lenard recursion sequence $\left\{g_{j}=\left(b_{j}, b_{j+1}\right)^{T} \mid j=\right.$ $-1,0,1, \cdots\}, b_{j}, b_{j+1}$ are all $u, v, u_{x}, v_{x}, \cdots$.

Theorem 5. If take $J g-1=0$, then can get double order eigenvalue problem (1) of the corresponding development equation family is:

$$
\left(\begin{array}{c}
v \\
u
\end{array}\right)_{t_{m}}=X_{m}=J g_{m}=K g_{m-1}, m=0,1,2, \cdots
$$

At the same time the development equation is compatibility conditions. for the family to Lax

$$
\left\{\begin{array}{l}
L \varphi=\lambda \varphi_{x} \\
\varphi t_{m}=w_{m} \varphi
\end{array} \quad m=0,1,2, \cdots\right.
$$

Note 2. Take $g_{-1}=\left(b_{-1}, b_{0}\right)^{T}=(0,1)^{T}$, get the development of family of equation (8) just is second order eigenvalue problem (1) Bargmann system corresponding development equation family. Here are the first three nontrivial development party path:

$$
\begin{aligned}
& \left(\begin{array}{l}
v \\
u
\end{array}\right)_{t_{0}}=K g_{-1}\left(\begin{array}{l}
v_{x} \\
u_{x}
\end{array}\right) \\
& \left(\begin{array}{l}
v \\
u
\end{array}\right)_{t_{1}}=K g_{0}\left(\begin{array}{c}
u_{x x x}+4 v u_{x}+2 u v_{x} \\
v_{x}+6 u u_{x}
\end{array}\right) \\
& \left(\begin{array}{l}
v \\
u
\end{array}\right)_{t_{2}}=K g_{1}\left(\begin{array}{l}
X_{11} \\
X_{21}
\end{array}\right)
\end{aligned}
$$

Among them:

$$
\begin{aligned}
X_{11}= & v_{x x x}+6 v v_{x}+18 u_{x} u_{x x}+6 u u_{x x x} \\
& +6 u^{2} v_{x}+24 u u_{x} v \\
X_{12}= & u_{x x x}+6 u v_{x}+6 v u_{x}+30 u^{2} u_{x}
\end{aligned}
$$

\section{Bargmann system and a set of reasonable coordinates on the Hamilton canonical form}

In order to study the structure and properties of the system, the system here is transformed into equivalent Hamilton system. This chapter first pass over the double Hamilton operator $\mathrm{K}$, J, functional gradient and Lenard recursion sequence determined grnan $\mathrm{Ba}$ article constraint parts, and getting the corresponding Ba rgmann system. Then use the constraint conditions of the order spectrum problem nonlinear change. And according to the Hamilton mechanics point of view and Euler - Lagrange equation thoughts, in the introduction of symplectic manifold $\left(w=\sum_{j=1}^{2} d y_{j} \wedge d z_{j}, R^{2 N}\right)$ to establish A set of appropriate coordinate, and in this basis and asked for BaTgman/n $J=1$ System equivalent Hamilton dynamic power system.

\subsection{Eigenvalue problem of Bargmann constraint and the corresponding system}

Put $\lambda_{1}\left\langle\lambda_{2}\left\langle\cdots\left\langle\lambda_{N}\right.\right.\right.$ for eigenvalue problem (2) of characteristic value, the $\varphi_{j}, \psi_{j}$ for corresponding to the characteristic value into the $\lambda_{j}(j=1,2, \cdots, N)$ of characteristic function. The following sign:

$$
\begin{aligned}
& \wedge=\operatorname{diag}\left(\lambda_{1}, \lambda_{2}, \cdots, \lambda_{N}\right), \\
& \Phi=\left(\varphi_{1}, \varphi_{2}, \cdots, \varphi_{N}\right)^{T}, \\
& \Psi=\left(\psi_{1}, \psi_{2}, \cdots, \psi_{N}\right)^{T},
\end{aligned}
$$

According to the (2) can get:

$$
\left\{\begin{array}{l}
L \Phi=\left(\partial^{2}+\Lambda u+v\right) \Phi=\Lambda \Phi_{x} \\
L \Psi=\left(\partial^{2}+\Lambda u+v\right) \Psi=-\Lambda \Psi_{x}
\end{array}\right.
$$


According to $K \nabla \lambda=J(\lambda \nabla \lambda)$, can get:

$$
K\left(\sum_{j=1}^{N} \lambda_{j}^{k} \nabla \lambda_{j}\right)=J\left(\sum_{j=1}^{N} \lambda_{j}^{k+1} \nabla \lambda_{j}\right), k=0,1,2, \cdots
$$

Is that to say:

$$
K\left(\begin{array}{c}
\left\langle\Lambda^{k} \Phi, \Psi\right\rangle \\
\left\langle\Lambda^{k+1} \Phi, \Psi\right\rangle
\end{array}\right)=J\left(\begin{array}{c}
\left\langle\Lambda^{k+1} \Phi, \Psi\right\rangle \\
\left\langle\Lambda^{k+2} \Phi, \Psi\right\rangle
\end{array}\right), k=0,1,2, \cdots
$$

Here, $\langle *, *\rangle$ Said standard inner product

$$
\langle f, g\rangle==\sum_{j=1}^{N} f_{i} g_{i}
$$

among them $f=\left(f_{1}, f_{2}, \cdots, f_{N}\right), g=\left(g_{1}, g_{2}, \cdots, g_{N}\right)$.

If make $g_{-1}=(0,1)^{T}$, according to $J g_{j}=K g_{j-1}(j=$ $0,1,2, \cdots)$ can be obtained:

$$
g_{1}=\left(\begin{array}{c}
2 u \\
2 v+6 u^{2}
\end{array}\right)
$$

let

$$
g_{1}=\left(\begin{array}{c}
2 u \\
2 v+6 u^{2}
\end{array}\right)=\left(\begin{array}{c}
\langle\Phi, \Psi\rangle \\
\langle\Lambda \Phi, \Psi\rangle
\end{array}\right)
$$

Through the type (15), after calculation can get double order eigenvalue problem (1) potential function $u, v$ and the corresponding characteristic function $\Phi$ and $\Psi$ tuen has the following constraint relationships:

$$
\left(\begin{array}{c}
v \\
u
\end{array}\right)=\left(\begin{array}{c}
\frac{1}{2}\langle\Lambda \Phi, \Psi\rangle-\frac{3}{4}\langle\Phi, \Psi\rangle^{2} \\
\frac{1}{2}\langle\Phi, \Psi\rangle
\end{array}\right)
$$

According to type (7), (11), (15), can get

$$
g_{j}=\left(\begin{array}{c}
b_{j} \\
b_{j+1}
\end{array}\right)=\left(\begin{array}{c}
\left\langle\Lambda^{j-1} \Phi, \Psi\right\rangle \\
\left\langle\Lambda^{j} \Phi, \Psi\right\rangle
\end{array}\right), j=1,2,3, \cdots
$$

According to the BaTgmann constraint type (14) can take double order eigenvalue problem (1) into:

$$
\left\{\begin{array}{l}
\Phi_{x x}+\frac{1}{2} \Lambda\langle\Phi, \Psi\rangle \Phi+\frac{1}{2}\langle\Lambda \Phi, \Psi\rangle \Phi-\frac{3}{4}\langle\Phi, \Psi\rangle^{2} \Phi=\Lambda \Phi_{x} \\
\Psi_{x x}+\frac{1}{2} \Lambda\langle\Phi, \Psi\rangle \Psi+\frac{1}{2}\langle\Lambda \Phi, \Psi\rangle \Psi-\frac{3}{4}\langle\Phi, \Psi\rangle^{2} \Psi=\Lambda \Psi_{x}
\end{array}\right.
$$

This is the order eigenvalue problem (1) Baqmann constraint. Below will use the constraint conditions and the corresponding Baumann system. Because of this type is a Bafgmann constraint get, so will its called order eigenvalue problem (1) Bargmann system.

\subsection{Group reasonable coordinates and Hamilton canonical system}

The first definition Lagrange function

$$
\hat{I}=\int_{\Omega} I d x
$$

Here, Lagrange density $I$ is

$$
\begin{aligned}
I= & \frac{1}{4}\langle\Phi, \Psi\rangle^{3}-\frac{1}{2}\langle\Lambda \Phi, \Psi\rangle\langle\Phi, \Psi\rangle-\frac{1}{2}\left\langle\Lambda \Phi, \Psi_{x}\right\rangle \\
& +\frac{1}{2\left\langle\Lambda \Phi_{x}, \Psi\right\rangle}+\left\langle\Phi_{x}, \Psi_{x}\right\rangle
\end{aligned}
$$

Theorem 6. Second order eigenvalue problem (1) of the corresponding Bafgmann system type (16) is equivalent to the Euler - Lagrange equation:

$$
\left\{\begin{array}{l}
\frac{\delta \hat{I}}{\delta \Phi}=0 \\
\frac{\delta \hat{I}}{\delta \Psi}=0
\end{array}\right.
$$

Proof.

$$
\begin{aligned}
\frac{\delta \hat{I}}{\delta \Psi} & =\frac{\partial I}{\partial \Psi}-\left(\frac{\partial I}{\partial \Psi_{x}}\right)_{x} \\
& =-\Psi_{x x}-\frac{1}{2} \Lambda\langle\Phi, \Psi\rangle \Psi-\frac{1}{2}\langle\Lambda \Phi, \Psi\rangle \Psi \\
& +\frac{3}{4}\langle\Phi, \Psi\rangle^{2} \Psi-\Lambda \Psi_{x} \\
& =-\left(\bar{L} \Psi+\Lambda \Psi_{x}\right) \\
& =0
\end{aligned}
$$

Similarly available:

$$
\frac{\delta \hat{I}}{\delta \Psi}=-\left(L \Phi-\Lambda \Phi_{x}\right)=0
$$

Define Euler - Lagrange equation (19) of the generalized coordinates are as follows:

$$
u_{1}=\Phi, u_{2}=\Psi
$$

At the same time the need to find and generalized coordinates the corresponding generalized momentum $v_{1}, v_{2}$ and function $g$, make they constitute Hamilton system, namely to meet the following Hamilton canonical equation:

$$
\left\{\begin{array}{l}
u_{j x}=\left\{u_{j}, g\right\}=\frac{\partial g}{\partial v_{j}} \\
v_{j x}=\left\{v_{j}, g\right\}=-\frac{\partial g}{\partial u_{j}}
\end{array} \quad j=1,2 .\right.
$$

Among them, the $\{*, *\}$ says symplectic manifold $(w=$ $\left.\sum_{j=1}^{2} d y_{j} \wedge d z_{j}, R^{2 N}\right)$ even if $F, H$ for XinLiu Metaphysical Hamilton function, then

$$
\begin{aligned}
\{F, H\} & =\sum j=1^{2} \sum_{k=1}^{N}\left(\frac{\partial F}{\partial u_{j} k} \frac{\partial H}{\partial v_{j} k}-\frac{\partial F}{\partial v_{j} k} \frac{\partial H}{\partial u_{j} k}\right) \\
& =\sum_{j=1}^{2}\left(\left\langle F_{u_{j}}, H_{v_{j}}\right\rangle-\left\langle F_{v_{j}}, H_{u_{j}}\right\rangle\right)
\end{aligned}
$$


let

$$
g=\sum_{j=1}^{2}\left\langle u_{j x}, v_{j}\right\rangle-I
$$

obtain

$$
d g=\sum_{j=1}^{2}\left(\left\langle v_{j}, d u_{j x}\right\rangle+\left\langle u_{j x}, d v_{j}\right\rangle\right)-d I
$$

And by $g$ is $u_{j}, v_{j}$ function, so

$$
\begin{aligned}
d g & =\sum_{j=1}^{2}\left(\left\langle\frac{\partial g}{\partial u_{j}}, d u_{j}\right\rangle+\left\langle\frac{\partial g}{\partial v_{j}}, d v_{j}\right\rangle\right) \\
& =\sum_{j=1}^{2}\left(-\left\langle v_{j x} d u_{j}\right\rangle+\left\langle u_{j x}, d v_{j}\right\rangle\right)
\end{aligned}
$$

so

$$
\begin{aligned}
d I & =\left\langle d u_{1 x}, v_{1}\right\rangle+\left\langle v_{1 x}, d u_{1}\right\rangle+\left\langle d u_{2 x}, v_{2}\right\rangle+\left\langle v_{2 x}, d u_{2}\right\rangle \\
& =\left\langle d \Phi, v_{1 x}\right\rangle+\left\langle d \Phi_{x}, v_{1}\right\rangle+\left\langle d \Psi, v_{2 x}\right\rangle+\left\langle d \Psi_{x}, v_{2}\right\rangle
\end{aligned}
$$

so

$$
\left\{\begin{array}{l}
v_{1 x}=\frac{\partial I}{\partial \Phi} \\
v_{1}=\frac{\partial I}{\partial \Phi_{x}} \\
v_{2 x}=\frac{\partial I}{\partial \Psi} \\
v_{2}=\frac{\partial I}{\partial \Psi_{x}}
\end{array}\right.
$$

Thus get generalized momentum:

$$
v_{1}=\Psi_{x}+\frac{1}{2} \Lambda \Psi, v_{1}=\Phi_{x}-\frac{1}{2} \Lambda \Phi
$$

Theorem 7. Jacobi - Ostrogradsky coordinates

$$
\left\{\begin{array}{l}
u_{1}=\Phi \\
u_{2}=\Psi \\
v_{1}=\Psi_{x}+\frac{1}{2} \Lambda \Psi \\
v_{2}=\Phi_{x}-\frac{1}{2} \Lambda \Phi
\end{array}\right.
$$

Eigenvalue problem (1) BaTgmann system type (16) equivalent to Hamilton canonical system [13]:

$$
\left\{\begin{array}{l}
u_{j x}=\left\{u_{j}, g\right\}=\frac{\partial g}{\partial v_{j}} \\
v_{j x}=\left\{v_{j}, g\right\}=-\frac{\partial g}{\partial u_{j}}
\end{array} \quad j=1,2 .\right.
$$

Among them, Hamilton function $g$ is:

$$
\begin{aligned}
g= & -\frac{1}{2}\left\langle\Lambda v_{2}, u_{2}\right\rangle-\frac{1}{4}\left\langle\Lambda^{2} u_{1}, u_{2}\right\rangle+\left\langle v_{2}, v_{1}\right\rangle+\frac{1}{2}\left\langle\Lambda u_{1}, v_{1}\right\rangle \\
& -\frac{1}{4}\left\langle u_{1}, u_{2}\right\rangle^{3}+\frac{1}{2}\left\langle u_{1}, u_{2}\right\rangle\left\langle\Lambda u_{1}, u_{2}\right\rangle
\end{aligned}
$$

Proof. $u_{1}, u_{2}, v_{1}, v_{2}$ and Hamilton function $g$ substitution Hamilton equation:

$$
\left\{\begin{array}{l}
u_{j x}=\left\{u_{j}, g\right\}=\frac{\partial g}{\partial v_{j}} \\
v_{j x}=\left\{v_{j}, g\right\}=-\frac{\partial g}{\partial u_{j}}
\end{array} \quad j=1,2\right.
$$

Can be directly calculated with Bargmann system type (16) of equivalence.

Theorem 8. If take symplectic manifold coordinates for:

$$
\left\{\begin{array}{l}
y_{1}=\Phi \\
y_{2}=\Phi_{x}-\frac{1}{2} \Lambda \Phi \\
z_{1}=-\Psi_{x}-\frac{1}{2} \Lambda \Psi \\
z_{2}=\Psi
\end{array}\right.
$$

Eigenvalue problem (1) BaTgmann system type (16) equivalent to Hamilton canonical system [13]:

$$
\left\{\begin{array}{l}
\varphi_{j x}=\left\{\varphi_{j}, h\right\}=-\frac{\partial h}{\partial \psi_{j}} \\
\psi_{j x}=\left\{\psi_{j}, h\right\}=\frac{\partial h}{\partial \varphi_{j}}
\end{array} \quad j=1,2\right.
$$

Among them, Hamilton function $h$ is:

$$
\begin{aligned}
g= & -\frac{1}{2}\left\langle\Lambda y_{2}, z_{2}\right\rangle-\frac{1}{4}\left\langle\Lambda^{2} y_{1}, z_{2}\right\rangle-\left\langle y_{2}, z_{1}\right\rangle \\
& -\frac{1}{2}\left\langle\Lambda y_{1}, z_{1}\right\rangle-\frac{1}{4}\left\langle y_{1}, z_{2}\right\rangle^{3}+\frac{1}{2}\left\langle y_{1}, z_{2}\right\rangle\left\langle\Lambda y_{1}, z_{2}\right\rangle
\end{aligned}
$$

Note 3. According to (21), and potential function and characteristic function between the Bafgmann constraint type:

$$
\left(\begin{array}{c}
v \\
u
\end{array}\right)=\left(\begin{array}{c}
\frac{1}{2}\left\langle\Lambda y_{1}, z_{2}\right\rangle-\frac{3}{4}\left\langle y_{1}, z_{2}\right\rangle^{2} \\
\frac{1}{2}\left\langle y_{1}, z_{2}\right\rangle
\end{array}\right)
$$

\section{Bargmann under the restriction of the Hamilton equation and completely integrability}

In this chapter, first through the above the obtained Jacobi - Ostrogradsky coordinates, the energy is in accordance with the potential and in accordance with the speed second order eigenvalue problem of operator pedigree into the form of matrix, the nonlinear change. And then according to the potential function $u, v$ and characteristic function, the relationship between the development of the family will be equation solution into finite dimensional completely integrable system is, its essence is the use of finite dimensional Hamilton canonical system could be fully integrated solution for the soliton system solution.

\subsection{Bargmann under the restriction of Lax}

Theorem 9. Second order eigenvalue problem (1) corresponding BaTamann constraint system type (16) in the coordinate system type (21) equivalent to the matrix form:

$$
\left\{\begin{array}{l}
Y_{x}=M Y \\
Z_{x}=-M^{T} Z
\end{array}\right.
$$


Among them:

$$
\begin{aligned}
& Y=\left(\begin{array}{l}
y_{1} \\
y_{2}
\end{array}\right)=\left(\begin{array}{c}
\Phi \\
\Phi_{x}-\frac{1}{2} \Lambda \Phi
\end{array}\right) \\
& Z=\left(\begin{array}{c}
z_{1} \\
z_{2}
\end{array}\right)=\left(\begin{array}{c}
-\Psi_{x}-\frac{1}{2} \Lambda \Psi \\
\Psi
\end{array}\right) \\
& M=\left(\begin{array}{c}
E \\
\frac{1}{4} \Lambda^{2}-\Lambda u-v \frac{1}{2} \Lambda
\end{array}\right), \\
& E=E_{N * N}=\operatorname{diag}(1,1, \cdots, 1)
\end{aligned}
$$

Proof. Martial arts (26), (27) direct substitution type (25), and then know and type (16) is of equivalence.

Theorem 10. In the coordinate type (21) basis, with the second order eigenvalues problem (1) related auxiliary spectrum problem

$$
\begin{gathered}
\Phi_{t_{m}}=w_{m} \Phi=\sum_{j=0}^{m}\left(-\frac{1}{2} b_{j x}-\frac{1}{2} \Lambda b_{j}+b_{j} \partial\right) \Lambda^{m-j} \Phi \\
m=0,1,2, \cdots
\end{gathered}
$$

transformation:

$$
\begin{aligned}
& \left\{\begin{array}{l}
Y_{t_{m}}=V_{m} Y \\
Z_{t_{m}}=-V_{m}^{T} Z
\end{array} \quad m=0,1,2, \cdots\right. \\
& V_{m}=\left(\begin{array}{ll}
A_{m} & B_{m} \\
C_{m} & D_{m}
\end{array}\right) \\
& A_{m}=\sum_{j=0}^{m}-\frac{1}{2} b_{j x} \Lambda^{m-j} \\
& B_{m}=\sum_{j=0}^{m} b_{j} \Lambda^{m-j} \\
& C_{m}=\sum_{j=0}^{m}\left(-\frac{1}{2} b_{j x x}+\frac{1}{4} \Lambda^{2} b_{j}-b_{j} \Lambda u-b_{j} v\right) \Lambda^{m-j} \\
& D_{m}=\sum_{j=0}^{m} \frac{1}{2} b_{j x} \Lambda^{m-j}
\end{aligned}
$$

Corollary 1. In Bavgmam constraint type (24), and second order eigenvalue problem (1) related development equation family type (8) to write the Lax for:

$$
\begin{aligned}
& \left\{\begin{array}{l}
Y_{x}=M Y \\
Y_{t_{m}}=V_{m} Y
\end{array} \quad m=0,1,2, \cdots\right. \\
& \left\{\begin{array}{l}
Z_{x}=-M^{T} Z \\
Z_{t_{m}}=-V_{m}^{T} Z
\end{array} \quad m=0,1,2, \cdots\right.
\end{aligned}
$$

Among them:

$$
M=\left(\begin{array}{cc}
\frac{1}{2} \Lambda & E \\
\alpha & \frac{1}{2} \Lambda
\end{array}\right)
$$

Among

them: $\alpha=\frac{1}{4} \Lambda^{2}-\frac{1}{2} \Lambda\left\langle y_{1}, z_{2}\right\rangle-\frac{1}{2}\left\langle\Lambda y_{1}, z_{2}\right\rangle+\frac{3}{2}\left\langle y_{1}, z_{2}\right\rangle^{2}$ Here the $V_{m}$ of for:

$$
\begin{aligned}
A m= & \sum_{j=1}^{m}-\frac{1}{2}\left(\left\langle\Lambda^{j-1} y_{2}, z_{2}\right\rangle-\left\langle y_{1}, \Lambda^{j-1} z_{1}\right\rangle\right) \Lambda^{m-j} \\
B m= & \sum_{j=1}^{m}\left\langle\Lambda^{j-1} y_{1}, z_{2}\right\rangle \Lambda^{m-j}+\Lambda^{m} \\
C m= & \sum_{j=1}^{m}\left(-\frac{1}{4}\left\langle\Lambda^{j+1} y_{1}, z_{2}\right\rangle+\frac{1}{2}\left\langle y_{1}, z_{2}\right\rangle\left\langle\Lambda^{j} y_{1}, z_{2}\right\rangle\right. \\
& +\left\langle\Lambda^{j-1} y_{2}, z_{1}\right\rangle+\frac{1}{4}\left\langle\Lambda^{j-1} y_{1}, z_{2}\right\rangle \Lambda^{2}+ \\
& \left.-\frac{1}{2}\left\langle\Lambda^{j-1} y_{1}, z_{2}\right\rangle\left\langle y_{1}, z_{2}\right\rangle \Lambda\right) \lambda^{m-j}+\frac{1}{4} \lambda^{m+2} \\
& -\frac{1}{2}\left\langle y_{1}, z_{2}\right\rangle \Lambda^{m+1}-\frac{1}{2}\left\langle\Lambda y_{1}, z_{2}\right\rangle \Lambda^{m}+\frac{3}{4}\left\langle y_{1}, z_{2}\right\rangle^{2} \Lambda^{m} \\
D_{m}= & \sum_{j=1}^{m} \frac{1}{2}\left(\left\langle\Lambda^{j-1} y_{2}, z_{2}\right\rangle-\left\langle y_{1}, \Lambda^{j-1} z_{1}\right\rangle\right) \Lambda^{m-j}
\end{aligned}
$$

\section{Proof.}

$$
\left\{\begin{array}{l}
L \Phi=\Lambda \Phi_{x} \\
\Phi_{t_{m}}=w_{m} \Phi=\sum_{j=0}^{m}\left(-\frac{1}{2} b_{j x}-\frac{1}{2} \Lambda b_{j}+b_{j} \partial\right) \Lambda^{m-j} \Phi
\end{array}\right.
$$

\subsection{Hamilton equation of the family completely}

integrability and solution of the said

Theorem 11. In the symplectic space $\left(R^{2 N}, \sum_{j=1}^{2}\right.$ $\left.d y_{j} \wedge d z_{j}\right)$, the use of BaTgmann constraint type (24), can $J=I$ To get and second order eigenvalue problem (1) the corresponding development equation family type (8) Lax of polices, type (30), (31) is equivalent to the Hamilton canonical system:

$$
\left\{\begin{array}{l}
Y_{x}=-\frac{\partial h}{\partial Z} \\
Y_{t_{m}}=-\frac{\partial h_{m}}{\partial Z}
\end{array} \quad m=0,1,2, \cdots\right.
$$

and

$$
\left\{\begin{array}{l}
Z_{x}=\frac{\partial h}{\partial Y} \\
Z_{t_{m}}=\frac{\partial h_{m}}{\partial Y}
\end{array} \quad m=0,1,2, \cdots\right.
$$

Among them, the $h$ by type (23) said, $h_{m}$ [13] take for:

$$
\begin{aligned}
g= & -\frac{1}{2}\left\langle\Lambda^{m+1} y_{1}, z_{2}\right\rangle\left\langle y_{1}, z_{2}\right\rangle-\frac{1}{4}\left\langle\Lambda y_{1}, z_{2}\right\rangle\left\langle\Lambda^{m} y_{1}, z_{2}\right\rangle \\
& +\frac{1}{4}\left\langle y_{1}, z_{2}\right\rangle^{2}\left\langle\Lambda^{m} y_{1}, z_{2}\right\rangle+\frac{1}{4}\left\langle\Lambda^{m+2} y_{1}, z_{2}\right\rangle \\
& +\left\langle\Lambda^{m} y_{2}, z_{1}\right\rangle-\sum_{j=1}^{m}\left|\left\langle\Lambda^{m-j} y_{1}, z_{1}\right\rangle\left\langle\Lambda^{j-1} y_{1}, z_{2}\right\rangle\right| \\
\left\langle\Lambda_{2}, z_{1}\right\rangle\left\langle\Lambda^{j-1} y_{2}, z_{2}\right\rangle \mid & \\
& +\sum_{j=1}^{m} \frac{1}{4}\left(\left\langle\Lambda^{j-1} y_{1}, z_{1}\right\rangle\right. \\
& \left.+\left\langle\Lambda^{j-1} y_{2}, z_{2}\right\rangle\right)\left(\left\langle\Lambda^{m-j} y_{1}, z_{1}\right\rangle+\left\langle\Lambda^{m-j} y_{2}, z_{2}\right\rangle\right)
\end{aligned}
$$


Proof. In the coordinates (21) foundation, according to Lax to type (30), (31) and Hamilton equation of the structure characteristic, can get:

$$
\begin{aligned}
\frac{\partial h_{m}}{\partial z_{1}}= & \sum_{j=1}^{m}-\frac{1}{2}\left(\left\langle\Lambda^{j-1} y_{2}, z_{2}\right\rangle-\left\langle y_{1}, \Lambda^{j-1} z_{1}\right\rangle\right) \Lambda^{m-j} y_{1} \\
& +\sum_{j=1}^{m}\left\langle\Lambda^{j-1} y_{1}, z_{2}\right\rangle \Lambda^{m-j} y_{2}+\Lambda^{m} y_{2} \\
\frac{\partial h_{m}}{\partial z_{2}}= & \sum_{j=1}^{m}\left(-\frac{1}{4}\left\langle\Lambda^{j+1} y_{1}, z_{2}\right\rangle+\frac{1}{2}\left\langle y_{1}, z_{2}\right\rangle\left\langle\Lambda^{j} y_{1}, z_{2}\right\rangle\right. \\
& +\left\langle\Lambda^{j-1} y_{2}, z_{1}\right\rangle+\frac{1}{4}\left\langle\Lambda^{j-1} y_{1}, z_{2}\right\rangle \Lambda^{2} \\
& \left.-\frac{1}{2}\left\langle\Lambda^{j-1} y_{1}, z_{2}\right\rangle\left\langle y_{1}, z_{2}\right\rangle \Lambda\right) \Lambda^{m-j} y_{1}+\frac{1}{4} \Lambda^{m+2} y_{1} \\
& -\frac{1}{2}\left\langle y_{1}, z_{2}\right\rangle \Lambda^{m+1} y_{1}-\frac{1}{2} \Lambda\left\langle y_{1}, z_{2}\right\rangle \Lambda^{m} y_{1} \\
& +\frac{3}{4}\left\langle y_{1}, z_{2}\right\rangle^{2} \Lambda^{m} y_{1}+\sum_{j=1}^{m}\left(\frac{1}{2}\left\langle\Lambda^{j-1} y_{2}, z_{2}\right\rangle\right. \\
& \left.-\frac{1}{2}\left\langle y_{1}, \Lambda^{j-1} z_{1}\right\rangle\right) \Lambda^{m-j} y_{2}
\end{aligned}
$$

Using integral, the some items to piece together, through a lot of calculation can be obtained $h_{m}$.

So, infinite dimensional dynamic system was constraint condition constraint in the finite dimensional submanifold inside. Hamilton canonical system in the sense of Liouville complete integrability $[15,16]$ research is the a important content, the following we mainly discuss (33), (34) in the Liouville sense completely integrability. First of all take:

$$
\begin{aligned}
E_{k}^{(1)}= & y_{2 k} z_{1 k}+\frac{1}{4} \lambda_{k}^{2} y_{1 k} z_{2 k}+\frac{1}{4} y_{1 k} z_{2 k}\left\langle y_{1}, z_{2}\right\rangle^{2} \\
& -\frac{1}{4} y_{1 k} z_{2 k}\left\langle\Lambda y_{1}, z_{2}\right\rangle-\frac{1}{4} \lambda_{k} y_{1 k} z_{2 k}\left\langle y_{1}, z_{2}\right\rangle \\
& -\Gamma_{k} \\
E_{k}^{(2)}= & \sum_{l=1, l \neq k}^{N} \frac{1}{\lambda_{k}-\lambda_{l}} B_{i j}
\end{aligned}
$$

Using integral, the some items to piece together, through a lot of calculation can be obtained $h_{m}$.

Theorem 12. (1) $\left\{d E_{k}^{I}, I=1,2 ; K=1,2, \cdots, N\right\}$ each other for linear independence, And $\left\{E_{K}^{I}, I=1,2 ; k=1,2\right.$, $\cdots, N\}$ is convolution system, namely to meet:

$$
\left\{E_{k}^{I}, E_{j}^{J}\right\}=0, I, J=1,2 ; k, j=1,2, \cdots N .
$$

(2)

$$
\begin{aligned}
& h_{m}=\sum_{k=1}^{N} \lambda_{k}^{m} E_{k}^{I} \\
& \left\{h, E_{k}^{I}\right\}=0, \forall k=1,2, \cdots, N . I=1,2 . \\
& \left\{h, h_{m}\right\}=0, \forall m=1,2, \cdots \\
& \left\{h_{n}, h_{m}\right\}=0, \forall m, n=1,2, \cdots
\end{aligned}
$$

Using integral, the some items to piece together, through a lot of calculation can be obtained $h_{m}$. In the sense of Liouville, $h$ and $\left\{h_{m}, m=1,2, \cdots\right\}$ are completely integrable system.

Theorem holdings if $\left\{y_{j}, z_{j} \mid j=1,2\right\}$ for Hamilton canonical system type (33), (34) solution, then

$$
\left(\begin{array}{c}
v \\
u
\end{array}\right)=\left(\begin{array}{c}
\frac{1}{2}\left\langle\Lambda y_{1}, z_{2}\right\rangle-\frac{3}{4}\left\langle y_{1}, z_{2}\right\rangle^{2} \\
\frac{1}{2}\left\langle y_{1}, z_{2}\right\rangle
\end{array}\right)
$$

Using integral, the some items to piece together, through a lot of calculation can be obtained $h_{m}$. Is second order eigenvalue problem (1) of the corresponding development equation family type (8) solution [17], is type (8) solution to GeBiao and of [18]. According to the Hamilton canonical system type (31), (4 to 8 ) and the corresponding equation of the family to Lax of equivalence and integrability, known and order eigenvalue problem (1) related development equation family type (8) solution can through the symplectic space $\left(R^{2 N}, \mathrm{E}\right.$ dy $\mathrm{A}$ DZJ) integrable system convolution solution was said. The fifth chapter conclusion This paper have given energy is in accordance with the potential and in accordance with the speed order eigenvalue problem and its corresponding $\mathrm{Ba}$ gmann system was studied, the main won the following conclusion:

(1) obtained and order eigenvalue problem related development equation family:

$$
\left(\begin{array}{c}
v \\
u
\end{array}\right)_{t_{m}}=X_{m}=J g_{m}=K g_{m-1}, m=0,1,2, \cdots
$$

$$
\left\{\begin{array}{l}
u=\frac{1}{2}\langle\Phi, \Psi\rangle \\
v=\frac{1}{2}\langle\Lambda \Phi, \Psi\rangle-\frac{3}{4}\langle\Phi, \Psi\rangle^{2}
\end{array}\right.
$$

(3) take Jacobi - Ostrogradsky coordinates for

$$
\left\{\begin{array}{l}
y_{1}=\Phi \\
y_{2}=\Phi_{x}-\frac{1}{2} \Lambda \Phi \\
z_{1}=-\Psi_{x}-\frac{1}{2} \Lambda \Psi \\
y_{2}=\Psi
\end{array}\right.
$$

Get the finite dimensional Hamilton canonical system:

$$
\left\{\begin{array} { l } 
{ Y _ { x } = - \frac { \partial h } { \partial Z } } \\
{ Y _ { t _ { m } } = - \frac { \partial h _ { m } } { \partial Z } }
\end{array} \quad \left\{\begin{array}{l}
Z_{x}=\frac{\partial h}{\partial Y} \\
Z_{t_{m}}=\frac{\partial h_{m}}{\partial Y}
\end{array} \quad m=0,1,2, \cdots\right.\right.
$$

(4) if $\left\{y_{j}, z_{j} \mid j=1,2\right\}$ for Hamilton canonical system

$$
\left\{\begin{array} { l } 
{ Y _ { x } = - \frac { \partial h } { \partial Z } } \\
{ Y _ { t _ { m } } = - \frac { \partial h _ { m } } { \partial Z } }
\end{array} \quad \left\{\begin{array}{l}
Z_{x}=\frac{\partial h}{\partial Y} \\
Z_{t_{m}}=\frac{\partial h_{m}}{\partial Y}
\end{array} \quad m=0,1,2, \cdots\right.\right.
$$

Is The family of the development equation (8)s solution. Today, we known that system is less, so looking for new integrable system, especially with practical significance of the equation related integrable system is mathematical physical society's full attention. With using the constraint relationship structure equation solution has also become a very important research field. 


\section{Acknowledgement}

The authors acknowledge the financial support ..., project No .... The author is grateful to the anonymous referee for a careful checking of the details and for helpful comments that improved this paper.

\section{References}

[1] ChenDeng Yuan. Soliton in. Beijing: science press, 1-12 (2006).

[2] V. I. Arnold. Mathematical Methods of Classical Mechanics. New York: Spring - Verlag New York Berlin Heidelberg, 5575 (1999).

[3] GuChaoHao. Soliton theory and application. Hangzhou: zhejiang science and technology press, 1-40 (1990).

[4] generation red English, WangZhongQing. Fiber soliton and optical soliton communication. Chongqing post and telecommunications university, 77-80 (2004).

[5] British o. Classical mechanics mathematical methods. Qi friend, translation. Beijing: higher education press, 165-220 (1992).

[6] C. W. Cao. Symplectic manifold and integrable system. Journal of Shijiazhuang Railway Institute, 1-9 (1989).

[7] C. W. Cao. A Classical Integrable System and the Involutive Representation of Solutions of the KDV Equation. Acta Mathematica Sinica, 216-223 (1991).

[8] A. Marek, P. F. Allan. Super - Extensions of Energy Dependent Schrodinger Operators. Com - mun. Math. Phys, 487-500 (1989).

[9] C. W. Cao, X. G. Geng, Y. T. Wu, From the special $2+1$ Toda lattice to the Kadomtsev - Petviashvili equation. J Math Phys, 8059-8078 (1999).

[10] QiaoZhiJun. Soliton family generation and transposition said general structure. Journal of applied mathematics, 287301 (1995).

[11] MaWenXiu. With the development of Lenard recursion structure equation of the family said Lax. Journal of mathematical physics, 409-418 (1994)

[12] LiYi god. Soliton and integrable system. Shanghai: Shanghai science and technology education press, 1-24 (1999).

[13] P. J. Olver. Applications of Lie group to differential equation. New York: Spring - Verlag New York Berlin Heidelberg, 13-53, 452-462 ( 1999).

[14] GuZhu full. A kind of new integrable system and its family of Mkdv equations show. Chinese science bulletin, 1-15 (1990).

[15] Z. Q. Gu, New Integrable Systems and the MKDV Hierarchy. Chinese Science Bulletin, 1-36 (1991).

[16] X. F. Ye, X. G. Geng, A General Generator of Involutive Systems and Finite - dimensional Integrable Systems. Xuzhou normal university press, 34-37 (2008).

[17] Z. Q. Gu, The Neumann system for The 3 rd - order eigenvalue problems related to The Boudssinesq equation. IL Nuove, 615-632 (2002).

[18] GengXian kingdom, cao CeWen. A Bargmann system and a kind of evolution equation solution of the convolution said. Mathematics yearbook, 92-98 (1992).

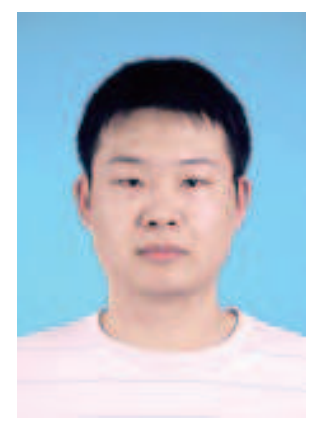

Sun Yinghui graduated from Yanshan University. His master's degree is from Tianjin University. Now he works in the School of Material Science and Engineering of Tianjin University. He participated in the five provincial-level research projects. $\mathrm{He}$ has published ten papers.

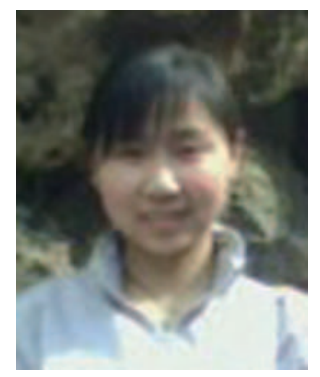

